\title{
On The Cauchy Problem For Some Parabolic Fractional Partial Differential Equations With Time Delays
}

\author{
Mahmoud M.El-Borai, Wagdy G.El-Sayed, Faez N. Ghaffoori \\ Department of Mathematics, Facultyof Science, Alexandria University, Alexandria Egypt
}

Received: February 17, 2016 / Accepted: March 16, 2016 / Published: May 25, 2016.

Abstract: The Cauchy problem for some parabolic fractional partial differential equation of higher orders and with time delays is considered. The existence and unique solution of this problem is studied. Some smoothness properties with respect to the parameters of these delay fractional differential equations are considered.

Keywords: Cauchy problem- fractional partial differential equations with time delays- successive approximations.

\section{Introduction}

We will adhere the following notations, $R^{n}$ is n-dimensional Euclidean space, $x, y$ are elements of this space, $q=\left(q_{1}, q_{2}, \ldots, q_{n}\right)$ is a multi-index,

$$
\begin{gathered}
D_{x}=\frac{\partial}{\partial x_{1}}, \frac{\partial}{\partial x_{2}}, \ldots, \frac{\partial}{\partial x_{n}} \\
D_{x}^{q}=\frac{\partial^{|q|}}{\partial x_{1} q_{1} \partial x_{2}{ }^{q_{2}} \cdots \partial x_{n}{ }^{q_{n}}} \\
|q|=q_{1}+q_{2}+\cdots+q_{n} \\
D_{t}=\frac{\partial}{\partial t},|x|^{2}=x_{1}^{2}+x_{2}^{2}+\cdots+x_{n}^{2}
\end{gathered}
$$

Consider the parabolic fractional partial differential equations with time delay of the form

$$
\begin{gathered}
D_{t}^{\alpha} u(x, t, p)+\sum_{|q| \leq 2 m} a_{q}(x, t) D_{x}^{q} u(x, t, p)= \\
\sum_{j=1}^{k} \sum_{|q|<2 m} b_{q j}(x, t) D_{x}^{q}\left(x, t-p_{j}, p\right)+h(x, t) \\
0<\alpha \leq 1,0<t \leq T, p=\left(p_{1}, \ldots, p_{k}\right), 0<p_{j}< \\
v<T, j=1,2, \ldots, k,
\end{gathered}
$$

where $v$ is a given positive number

Corresponding author: Mahmoud M.El-Borai, Department of Mathematics, Facultyof Science, Alexandria University, Alexandria, Egypt. E-mail: m_m_elborai@yahoo.com.
Let us suppose that $u$ satisfies the Cauchy condition,

$$
u(x, 0, p)=f(x)
$$

Where $f$ is a given function

Let $C^{r}(Q)$ denote the set of all functions defined in a neighborhood of each point of a domain $Q \subset R^{l}$, and having bounded continuous partial derivatives of all orders le-ss than or equal $\mathrm{r}$ in $Q(r$ denotes a nonnegative integer and $l$ a positive integer) .

We assume that $f \in C^{2 m-1}\left(R^{n}\right)$. Let $u$ satisfy

$$
\begin{aligned}
& u(x, t, p)=F(x, t),(x, t) \in Q_{1} \\
Q_{1}= & \left\{(x, t): x \in R^{n},-v<t<0,0<p_{j}<v\right\}
\end{aligned}
$$

Where $\mathrm{F}$ is a given bounded continuous function on $Q_{1}$.

Assume that $\mathrm{F} \in C^{2 m-1}\left(R^{n}\right)$ for every $t \in(-v, 0)$, and

$$
F(x, 0)=f(x), x \in R^{n}
$$

The linear partial differential operator $\sum_{|q|=2 m} a_{q}(x, t) D_{x}^{q}$ is supposed to be uniform elliptic on $R^{n}$, i.e. there exists a positive number $\lambda$ such that for every

$$
(x, t) \in Q_{2}=\left\{(x, t): x \in R^{n}, 0 \leq t \leq T\right\},
$$

and for every $y \in R^{n}$,

$$
(-1)^{m} \sum_{|q|=2 m} a_{q}(x, t) y^{q} \geq \lambda|y|^{2 m}
$$


Where the number $\lambda$ is independent of $x, t$ and $y$. It is suitable to rewrite the problem (1.1), (1.2) in the form

$$
\begin{gathered}
u(x, t, p)=f(x)+ \\
\frac{1}{\Gamma(\alpha)} \int_{0}^{t}(t-\eta)^{\alpha-1} \sum_{|q| \leq 2 m} a_{q}(x, t) D_{x}^{q} u(x, \eta, p) d \eta= \\
\frac{1}{\Gamma(\alpha)} \int_{0}^{t}(t-\eta)^{\alpha-1} \sum_{j=1}^{k} \sum_{|q|<2 m} b_{q j}(x, \eta) D_{x}^{q} u(x, \eta \\
\left.-p_{j}, p\right) d \eta
\end{gathered}
$$

Where $\Gamma$ is Gamma function

Following Friedman [11], we assume the following conditions.

$\left(C_{1}\right)$ All the coefficients $a_{q},|q| \leq 2 m$, are continuous and bounded functions on $Q_{2} \cdot\left(C_{2}\right)$ All the coefficients $a_{q},|q| \leq 2 m$, satisfy a Holder condition with respect to uniformly in $Q_{2}$, namely there exists a positive constant $\mathrm{K}$ and a constant $\gamma \in(0,1)$ such that

$$
\begin{gathered}
\left|a_{q}(x, t)-a_{q}(y, t)\right| \leq K|x-y|^{\gamma}, \text { for all } \\
x, y \in R^{n}, t \in[0, T]
\end{gathered}
$$

$\left(C_{3}\right)$ The coefficients $a_{q},|q|=2 m$, of the principle term are uniformly continuous as functions of $t$ relative to $(x, t)$ in $Q_{2}$.

$$
\left(C_{4}\right) D_{x}^{\beta} a_{q} \in C\left(Q_{2}\right) \quad, \quad \beta=\left(\beta_{1}, \ldots, \beta_{n}\right) \quad \text { is } \quad \text { a }
$$

multi-index $|\beta| \leq 2 m,|q| \leq 2 m$, and

$C\left(Q_{2}\right)$ is the set of all continuous bounded functions on $Q_{2}$.

We assume also that coefficients $b_{q j},|q| \leq 2 m$, lative to iple term are uniformly continuous as functions of $j=1,2, \ldots, k$, satisfy a Holder condition with respect to $x$, (similar to cond. $\left(C_{2}\right)$ ) and $D_{x}^{\beta} b_{q j} \in C\left(Q_{2}\right),|\beta|<2 m$.

In section 2, we study the existence and uniqueness of the solution of the Cauchy problem (1.1), (1.2). In section 3 , we consider the dependence of the parameters $p_{1}, \ldots, p_{k}$ of the solution of the considerd
Cauchy problem, compare [1], [10], [13], and [14].

\section{Uniqueness and Existence Theorems}

Consider the Cauchy problem

$$
\begin{gathered}
D_{t}^{\alpha} w(x, t)+\sum_{|q| \leq 2 m} a_{q}(x, t) D_{x}^{q} w(x, t)=0 \\
w(x, 0)=f(x)
\end{gathered}
$$

It is well known under the conditions $\left(C_{1}\right)-\left(C_{4}\right)$ that the solution of the Cauchy problem (2.1), (2.2) represented in the form [7]

$$
w(x, t)=\int_{0}^{\infty} \int_{-\infty}^{\infty} G\left(x-y, t^{\alpha} \theta\right) f(y) \zeta_{\alpha}(\theta) d y d \theta
$$

$\zeta_{\alpha}(\theta)$ is a probability density function define on $(0, \infty)$

$\mathrm{G}$ is the fundamental solution of equation (2.1), when $\alpha=1$, which has the following conditions [11].

$$
\begin{gathered}
\left(C_{5}\right) D_{x}^{q} G, D_{y}^{q} G, D_{t} G \in\left(Q_{2} \times Q_{2}\right),|q| \leq 2 m \\
\left(C_{6}\right)\left|D_{x}^{q} D_{y}^{B} G(x, t, y, \theta)\right| \leq \frac{K z(x-y, t-\theta)}{(t-\theta)^{v_{1}}}
\end{gathered}
$$

Where $z(x-y, t-\theta)=\exp \left[-v_{2}\left(|x-y|^{2 m} / t-\right.\right.$ $\left.\theta)^{\frac{1}{2 m-1}}\right],(0<\theta<t)$

$v_{1}=1 / 2 m[|q|+|\beta|+n], K, v_{2}$ are positive constants and $|q|<2 m,|\beta| \leq 2 m$.

$$
\begin{gathered}
\left(C_{7}\right)\left|D_{x}^{q}[G(x, t, y, \theta)-G(z, t, y, \theta)]\right| \\
\leq \frac{K|x-z|^{v_{3}}}{(t-\theta)^{v_{4}}} \Lambda,
\end{gathered}
$$

Where $\quad \Lambda=\exp \left[-v_{5}\left(|x-y|^{2 m} / t-\theta\right)^{\frac{1}{2 m}}\right]+$ $\exp \left[-v_{5}\left(|z-y|^{2 m} / t-\theta\right)^{\frac{1}{2 m}}\right]$,

$v_{4}=1 / 2 m\left(|q|+v_{3}+n\right), v_{3}$ is an arbitrary positive number $\leq 1, K$ and $v_{5}$ are positive constants and $|q|<2 m, 0<\theta<t$.

$$
\begin{array}{r}
\text { Let } \mathrm{P}=\left\{p \in R^{k}: 0<p_{i}<v, i=1, \ldots, k\right\} \\
Q_{3}=\left\{(x, t): x \in R^{n},-v<t<T\right\}
\end{array}
$$

We prove now the following uniqueness theorem.

Theorem 2.1: Let $u$ be a function defined on $Q_{3} \times$ $P$. Let $D_{t} u, D_{x}^{q} G \epsilon C\left(Q_{3}\right),|q| \leq 2 m$, for every 
fixed $p \in P$. Let $F=0$ on $Q_{1}$. If $u$ is a solution of the Cauchy problem (1.1), (1.2) on $Q_{2}$, which satisfies the condition (1.3) on $Q_{1}$. Then the solution is unique

Proof: We set $(x, 0, p)=0, h(x, t)=0, x \in R^{n}$, $p \in P, t>0$.

In this case we must prove $u(x, t, p)=0$, for sufficiently small $t>0, x \in R^{n}, p \in P$

Using $\left(C_{5}\right),\left(C_{6}\right)$ of the fundamental solution $G$, we get

$$
\begin{gathered}
\int_{-\infty}^{\infty} G(x, t, y, \theta) D_{y}^{q} u(y, \theta, p) d y= \\
\int_{-\infty}^{\infty}(-1)^{|q|} u(y, \theta, p) D_{y}^{q} G(x, t, y, \theta) d y
\end{gathered}
$$

And so there exists a number $c \epsilon(0,1)$ such that $\sup _{x}\left|\int_{-\infty}^{\infty} G(x, t, y, \theta) D_{y}^{q} u(y, \theta, p) d y\right| \leq \frac{K}{(t-\theta)^{c}}\|u\|$, $t>\theta$

Where $\|u\|=\sup _{x}|u(x, t, p)|,|q|<2 m, K$ is a positive constant. According to

the conditions imposed on the considered problem, we can write

$$
\begin{gathered}
u(x, t, p)= \\
\alpha \sum_{j=1}^{k} \sum_{|q|<2 m} \int_{\eta_{j}(t)}^{t} \int_{0}^{\infty} \int_{-\infty}^{\infty} \theta \zeta_{\alpha}(\theta)(t-\eta)^{\alpha-1} G(x \\
\left.-y,(t-\eta)^{\alpha} \theta\right) \\
\times b_{q j}(y, \eta) D_{y}^{q} u\left(y, \eta-p_{j}, p\right) d y d \theta d \eta
\end{gathered}
$$

Where

$$
\eta_{j}(t)=\left\{\begin{array}{cc}
t & t \leq p_{j} \\
p_{j} & t>p_{j}
\end{array}\right.
$$

Thus

$$
\begin{gathered}
u(x, t, p)= \\
\alpha \sum_{j=1}^{k} \sum_{|q|<2} \int_{\eta_{j}(t)}^{t} \int_{0}^{\infty} \int_{-\infty}^{\infty}(-1)^{|q|} u(y, \eta \\
\left.-p_{j}, p\right) \theta \zeta(\theta)(t-\eta)^{\alpha-1} \\
\times D_{y}^{q}\left[b_{q j}(y, \eta) G\left(x-y,(t-\eta)^{\alpha} \theta\right)\right] d y d \theta d \eta
\end{gathered}
$$

Since $D_{x}^{q} b_{q j} \in C\left(Q_{2}\right),|q|<2 m, j=1, \ldots, k$. It follows by using (2.4) that

$$
\begin{gathered}
g(t, p) \leq \alpha k \sum_{j=1}^{k} \int_{\eta_{j}(t)}^{t} \int_{0}^{\infty} \theta(t-\eta)^{-c \alpha} \theta^{-c}(t \\
-\eta)^{\alpha-1} \zeta_{\alpha}(\theta) g\left(\eta-p_{j}, p\right) d \theta d \eta \\
\leq \alpha k \sum_{j=1}^{k} \int_{\eta_{j}(t)}^{t}\left[\int_{0}^{\infty} \theta^{1-c} \zeta_{\alpha}(\theta) d \theta\right](t-\eta)^{(1-c) \alpha-1} g(\eta \\
\left.-p_{j}, p\right) d \eta
\end{gathered}
$$

where

$$
g(t, p)=\|u\|=\sup _{x}|u(x, t, p)|
$$

Thus

$$
\begin{array}{r}
\xi(t) \leq \frac{\alpha K}{\Gamma((1-c) \alpha+1)} \sum_{j=1}^{k} \int_{0}^{t-p_{j}}(t-\eta \\
\left.-p_{j}\right)^{(1-c) \alpha-1} \xi(\eta) d \eta
\end{array}
$$

for

$$
t>p_{j}
$$

Where $\xi(t)=\sup _{p} g(t, p)$

If $t \leq p_{j}, j=1,2, \ldots k$, there is nothing to prove. Suppose $t>p_{j}$, then from equation (2.5)

$$
\xi(t) \leq \frac{\alpha K}{\Gamma((1-c) \alpha+1)} \int_{0}^{t}(t-\eta)^{(1-c) \alpha-1} \xi(\eta) d \eta
$$

It is easy to get:

$$
\xi(t) \leq \frac{K^{*} M^{n} t^{n \delta}[\Gamma(\delta)]^{n-1}}{\Gamma(n \delta+1)}
$$

for all

$$
n=1,2, \ldots
$$

Where $M=\sup _{t} \xi(t), \quad \delta=(1-c) \alpha, K^{*}$ is a positive integer, letting $\rightarrow \infty$, we get

$$
\xi(t) \equiv 0 \text {, for all } t \text {. }
$$

We prove now the following existence theorem.

Theorem 2.2: There exists a unique function $u$,

$$
u, D_{t} u, D^{q} u \in C\left(Q_{3}\right),|q| \leq 2 m
$$

Such that $u$ represents the unique solution of the Cauchy problem (1.1), (1.2) on $Q_{2}$, which satisfies the condition (1.3) on $Q_{1}$.

Proof: Let us try to find the solution of the integro-partial differential equation.

$$
u=H+E u
$$

Where $\mathrm{H}$ is a given function defined on $Q_{2}$ by, 


$$
\begin{gathered}
H(x, t, p)=\int_{0}^{\infty} \int_{-\infty}^{\infty} G\left(x, y, t^{\alpha} \theta\right) f(y) \zeta_{\alpha}(\theta) d y d \theta+ \\
\alpha \int_{0}^{t} \int_{0}^{\infty} \int_{-\infty}^{\infty} \theta \zeta_{\alpha}(\theta)(t-\eta)^{\alpha-1} G(x \\
\left.-y,(t-\eta)^{\alpha} \theta\right) h(y, \eta) d y d \theta d \eta+ \\
\alpha \sum_{j=1}^{k} \sum_{|q|<2 m} \int_{0}^{\eta_{j}(t)} \int_{0}^{\infty} \int_{-\infty}^{\infty}(-1)^{|q|} F(y, \eta \\
\times D_{y}^{q}\left[b_{q j}(y, \eta) G\left(x-y,(t-\eta)^{\alpha} \theta\right)\right] d y d \theta d \eta
\end{gathered}
$$

And $\mathrm{E}$ is an integro-partial differential difference operator defined on $C\left(Q_{2}\right)$ by $E u=u^{*}$,

$$
\begin{gathered}
u^{*}(x, t, p)=\alpha \sum_{j=1}^{k} \sum_{|q|<2 m} \int_{\eta_{j}(t)}^{t} \int_{0}^{\infty} \int_{-\infty}^{\infty}(-1)^{|q|} u(y, \eta \\
\left.-p_{j}, p\right) \theta \zeta(\theta)(t-\eta)^{\alpha-1} \\
\times D_{y}^{q}\left[b_{q j}(y, \eta) G\left(x-y,(t-\eta)^{\alpha} \theta\right)\right] d y d \theta d \eta
\end{gathered}
$$

We apply the method of successive approximation to solve (2.7), to do this, set

$$
u_{r+1}=H+E u_{r}, \quad r=1,2, \ldots
$$

Where the zero approximation $u_{0}$ is taken to be zero. The linearity of the operator $E$ leads to the following equation

$$
u_{r+1}-u_{r}=E\left(u_{r}-u_{r-1}\right)
$$

using the conditions imposed on the coefficients and the properties of the fundamental solution $G$ of equation (2.2), we get by a similar method of the proof of theorem (2.1)

$$
\left\|\mathrm{u}_{\mathrm{r}+1}-\mathrm{u}_{\mathrm{r}}\right\| \leq \frac{K t^{\delta r}[\Gamma(\delta)]^{r-1}}{\Gamma(\delta r+1)}
$$

where $K$ is a positive constant, $\delta=(1-c) \alpha$.

Thus the required solution of equation (2.6) is given by

$$
u=\sum_{r=1}^{\infty}\left(u_{r}-u_{r-1}\right)
$$

This series is absolutely and uniformly convergent on $Q_{2}$ to the function $u \in C\left(Q_{2}\right)$.

To prove the smoothness of the function $u$, we consider the following equation

$$
V_{i}=D_{x_{i}} H+E u_{i}+P_{i} V_{i}
$$

Where

$$
\begin{gathered}
E u_{i}=u_{i}^{*}, P_{i} V_{i}=V_{i}^{*}, \\
u_{i}^{*}(x, t, p)=\alpha \sum_{j=1}^{k} \int_{\eta_{j}(t)}^{t} \int_{0}^{\infty} \int_{-\infty}^{\infty} \theta \zeta_{\alpha}(\theta)(t \\
-\eta)^{\alpha-1} b_{0, \ldots, 0, j}(y, \eta) u\left(y, \eta-p_{j}, p\right) \\
\times D_{x_{i}}\left[G\left(x-y,(t-\eta)^{\alpha} \theta\right)\right] d y d \theta d \eta \\
V_{i}^{*}(x, t, p) \\
=\alpha \sum_{j=1}^{k} \sum_{1 \leq|q| \leq 2 \mathrm{~m}} \int_{\eta_{j}(t)}^{t} \int_{0}^{\infty} \int_{-\infty}^{\infty}(-1)^{|q|-1} V_{i}(y, \eta \\
\left.-p_{j}, p\right) \theta \zeta(\theta){ }_{-y}(t-\eta)^{\alpha-1} D_{x_{i}} D_{y}^{q-e_{i}}\left[b_{q j}(y, \eta) G(x\right. \\
\left.\left.-y,(t-\eta)^{\alpha} \theta\right)\right] d y d \theta d \eta \\
=\frac{\partial}{\partial x_{i}}, e_{1}=(1,0, \ldots, 0), e_{2}=(0,1, \ldots, 0), e_{n} \\
=(0,0, \ldots, 1)
\end{gathered}
$$

are the unit vectors

If the solution $V_{i}$ of equation(2.7) exists in the space $C\left(Q_{2}\right)$, then the partial derivative $D_{x_{i}} u$ exists for every $i=1, \ldots, n$ and

$$
V_{i}=D_{x_{i}} u \in C\left(Q_{2}\right)
$$

Using a similar technique to that used to prove the existence of solution of equation (2.6) in the space $C\left(Q_{2}\right)$ and noting that $u$ is a known function in the space $C\left(Q_{2}\right)$ and using the conditions $\left(C_{5}\right)$ and $\left(C_{6}\right)$ of the function $\mathrm{G}$, we deduce that equation (2.7) has a unique solution $V_{i}$ in the space $C\left(Q_{2}\right)$, for every $i=1, \ldots, n$.

And so $V_{i}=D_{x_{i}} u \in C\left(Q_{2}\right)$, compare [2]

By induction we can prove that $D_{x_{i}}^{q} u \in C\left(Q_{2}\right)$ for $|q|<2 m$.

Now to prove that $D_{x}^{q} \in C\left(Q_{2}\right)$ for $|q|=2 m$, we need to prove first all the partial derivatives $D_{x}^{q} u$ satisfy for $|q|<2 m$, a Holder continuity condition with respect to $x$.

To do this, we notice that

$$
\begin{aligned}
D_{x}^{\beta} u(x, t, p)-D_{z}^{\beta} u(z, t, p) \\
\quad=D_{x}^{\beta} H(x, t, p)-D_{z}^{\beta} H(z, t, p)+
\end{aligned}
$$




$$
\begin{gathered}
\sum_{j=1}^{k} \sum_{|q|<2 m} \int_{\eta_{j}(t)}^{t} \int_{0}^{\infty} \int_{-\infty}^{\infty} \theta \zeta(\theta)(t-\eta)^{\alpha-1} b_{q j}(y, \eta) \\
\times D_{y}^{q} u(y, \eta, p)\left[D_{x}^{\beta} G\left(x-y,(t-\eta)^{\alpha} \theta\right)-D_{z}^{\beta} G(z-\right. \\
\left.\left.y,(t-\eta)^{\alpha} \theta\right)\right] d y d \theta d \eta
\end{gathered}
$$

Where $|\beta|<2 m$

Using condition $\left(C_{7}\right)$ of the function $\mathrm{G}$ and remembering the conditions imposed on the coefficients of equation (1.1) we can prove that there is a constant $\gamma \in(0,1)$ such that

$$
\begin{gathered}
\left|D_{x}^{\beta} u(x, t, p)-D_{z}^{\beta} u(z, t, p)\right| \leq \mathrm{K}|x-z|^{\gamma} \text { for all } \\
x, z \in R^{n}
\end{gathered}
$$

Where the positive constant $\mathrm{K}$, is independent of $x, z$ and $t$.

This completes the proof of the theorem.

\section{Properties of Smoothness}

In this section we consider the dependence on the parameters $p_{1}, \ldots, p_{k}$. We shall deduce some smoothness properties of the solution $u(x, t, p)$ with respect to $p$, compare [10].

Theorem 3.1: If $F$ has continuous bounded derivatives $\boldsymbol{D}_{\boldsymbol{t}}^{\boldsymbol{r}} \boldsymbol{F}$ on $\boldsymbol{Q}_{\mathbf{1}}$, then $u$ has continuous bounded derivatives

$$
D_{p_{i}}^{r} \text { u on } Q_{2} \times P, D_{p_{i}}=\frac{\partial}{\partial p_{i}}, r=1,2, \ldots, i=1, \ldots, k
$$

Proof: For a fixed $t \in(0, T]$ and a fixed $x \in R^{n}$, we consider the following equation

$$
\begin{aligned}
& \quad D_{t}^{\alpha} V_{r_{i}}(x, t, p)+\sum_{|q| \leq 2 m} a_{q}(x, t) D_{x}^{q} V_{r_{i}}(x, t, p) \\
& =\sum_{j=1}^{k} \sum_{|q|<2 m} b_{q j}(x, t) D_{x}^{q} V_{r_{i}}\left(x, t-p_{j}, p\right) \\
& \text { Where } p \epsilon P, \\
& V_{r_{i}}\left(x, t-p_{j}, p\right)= \\
& \left\{\begin{array}{c}
D_{p_{i}}^{r} F\left(x, t-p_{i}\right), p_{i} \geq t, i=j \quad \text { for } \quad \text { some } \quad \text { fixed } \\
0, p_{i} \geq t, i \neq j
\end{array}\right. \\
& i=1, \ldots, k
\end{aligned}
$$

Assume that

$$
V_{r_{i}}(x, 0, p)=0
$$

Equation (3.1) can be obtained from formally from equation (1.1) by applying formally the partial differential operator $D_{p_{i}}^{r}$.

If the Cauchy problem $(3.1),(3,2)$ has a solution, then we deduce immediately that

$$
D_{p_{i}}^{r} u=V_{r_{i}} \in C\left(Q_{2} \times P\right) \text {. }
$$

The Cauchy problem (3.1), (3.2) can be transformed to

$$
\begin{gathered}
V_{r_{i}}(x, t, p)=\alpha \sum_{|q|<2 m} \int_{0}^{\eta_{j}(t)} \int_{0}^{\infty} \int_{-\infty}^{\infty}(-1)^{|q|} D_{p_{i}}^{r} F(y, \eta \\
\left.\quad-p_{i}\right) \theta \zeta_{\alpha}(\theta)(t-\eta)^{\alpha-1} \\
\times D_{y}^{q}\left[b_{q_{i}}(y, \eta) G\left(x-y,(t-\eta)^{\alpha} \theta\right)\right] d y d \theta d \eta \\
+\alpha \sum_{j=1}^{k} \sum_{|q| \leq 2 \mathrm{~m}} \int_{\eta_{j}(t)}^{t} \int_{0}^{\infty} \int_{-\infty}^{\infty}(-1)^{|q|} V_{r_{i}}(y, \eta- \\
\left.p_{j}, p\right) \theta \zeta_{\alpha}(\theta)(t-\eta)^{\alpha-1} \times D_{y}^{q}\left[b_{q_{i}}(y, \eta) G(x-\right. \\
\left.\left.y,(t-\eta)^{\alpha} \theta\right)\right] d y d \theta d \eta
\end{gathered}
$$

Similar to theorem (2.2) we see that equation (3.3) satisfies the required. It can be also proved that

$$
\frac{\partial u(x, t, p)}{\partial p_{i} \partial p_{j}}=0, \text { for } i \neq j
$$

\section{References}

[1] H.T. Bank and P. I. Daniel, Estimation of delays and other parameter in nonlinear functional equations. Siam J. control. Optima. 21, 893-951(1993).

[2] M. M. El-Borai, on the initial value problem for a partial differential equation with operator coefficients, int. J. math. And math. Sci.Vol. 3 No. 1, 103-111 (1980).

[3] M. M. El-Borai, some probability densities and fundamental solutions of fractional evolution equation 14 , 433-440 (2002).

[4] M. M. El-Borai, the fundamental solutions for fractional evolution equation of pa- rabolic type, J. of Appl. Math. Stochastic Analysis (JAMSA) 2004, 199-211.

[5] M. M. El-Borai, K. El-Said El-Nadi and I. G, El-Akabawi. On some integro-diffe- rential equations of fractional orders, the International J. of contemporary Mathem atics, Vol. 1, 2006, No. 15, 719-726.

[6] M. M. El-Borai, Khairia El-Said El-Nadi, Iman G. El-Akabawy. Approximate sol- utions for nonlinear fractional heat equations. Int. j.Contemp. Math.Sciences 2007, 2 (27): 1307-1316.

[7] M. M. El-Borai, Khairia El-Said El-Nadi, Hoda A. Foad. On some fractional stoc- hastic delay differential equations. Computers and Mathematics with Applications 2010, 59: 1165-1170. 
[8] M. M. El-Borai, Khairia El-Said El-Nadi, Iman G. El-Akabawy. On some fractio- nal evolution equations. Computers and Mathematics with Applications 2010 59: 1352-1355.

[9] D.W.Dennis, J. A. Burns, and E. M.Cliff, Parameter identification for an abstract Cauchy problem by quasi-linearization.Quarterly of applied Mathematics, Vol. LI March, 1-22 (1993).

[10] A. Friedmann, partial differential equations of parabolic type, prentice-Hall, INC. Englewood cliffs, N. J. (1964).

[11] R.H. Martin and H. L. Smith, Reaction-diffusion systems with time delays ;mon- otonicity invariance. Comparison and convergence, J. Reine Angew Math. 1-35.(1991)

[12] K. Yoshida, the Hopf bifurcation and its stability for semi linear differential equ-ations with time delay arising in ecology. Hiroshima Math. J. 12, 321-348 (1982).

[13] Khairia El-Said El-Nadi, Mahmoud. M. El-Borai, Integrated Semi groups and $\mathrm{C}$-auchy problem for some fractional abstract differential equations. Life Science J-ournal. 2013 10(3) 793-798.

[14] L. Xuedong and W.H. Joseph, Center manifolds for partial differential equations with delay, proc. of Royal Society of Edinburgh, 122 A, 237-254 (1992). 